\title{
MIMO OFDM Over Underwater Acoustic Channels
}

\author{
Milica Stojanovic Northeastern University \\ Email: millitsa@ece.neu.edu
}

\begin{abstract}
MIMO OFDM communication is considered for spatial multiplexing of independent data streams over bandlimited, frequency-selective underwater acoustic channels. Long acoustic multipath, however, limits the applicability of MIMO channel estimation methods that require inversion of a matrix whose size is proportional to both the number of transmit elements and the multipath spread. To overcome this problem, an adaptive algorithm is used that does not require matrix inversion and operates in a decision-directed manner, thus reducing both the computational complexity and the overhead. The algorithm makes use of a phase synchronization method to compensate for the non-uniform Doppler shifting in a wideband acoustic system, and exploits the remaining temporal coherence. System performance is successfully demonstrated using real data transmitted over $1 \mathrm{~km}$ in shallow water, with a varying number of carriers (128-1024), transmitters (1-3), and modulation levels (4 and 8 PSK) in the 8-18 $\mathrm{kHz}$ band.
\end{abstract}

\section{INTRODUCTION}

Orthogonal frequency division multiplexing (OFDM) is considered for the next generation of acoustic modems as a low-complexity alternative to single-carrier modulation. The quest for efficient use of acoustic bandwidth pushes the system design towards a large number of carriers and multiple-input multiple-output (MIMO) configurations that support parallel transmission of independent data streams.

Acoustic bandwidth is fundamentally limited by sound absorption, as well as by transducer technology. For example, a $10 \mathrm{kHz}$ bandwidth may be available for transmission over $1 \mathrm{~km}$. The channel is further characterized by extended multipath (tens of milliseconds), while inevitable motion causes severe Doppler distortion at the low speed of sound (nominally 1500 $\mathrm{m} / \mathrm{s}$ ). Although the operational bandwidth may be limited to only a few $\mathrm{kHz}$, it is not negligible with respect to the center frequency-on the contrary, the two may be comparable. An acoustic system is thus a truly wideband system, in which the Doppler distortion (frequency shifting) is not uniform across the signal bandwidth.

Recent work has focused on demonstrating the viability of OFDM over acoustic channels. Two approaches have been pursued: one based on the classical principles of pilotassisted, block-oriented detection [1], [2], and another based on decision-directed, adaptive block processing [3]-[6]. The latter approach relies on Doppler tracking and phase prediction to provide reliable symbol decisions, which in turn enable reduction in the pilot overhead, and can also lead to an improved performance. In the present treatment, we make use of this approach in a MIMO system configuration.

This work was supported by the ONR MURI Grant \#N00014-07-1-0738 and the ONR grant N00014-07-1-0202.
In a MIMO OFDM system operating with $M_{T}$ transmit and $M_{R}$ receive elements, there are $M_{T} M_{R}$ channels whose transfer functions need to be estimated at each of the $K$ carriers. If performed in the impulse response domain, channel estimation will require $L<K$ coefficients per transmitter/receiver pair in a bandwidth-efficient acoustic system. To this end, at least $M_{T} L$ carriers have to contain known symbols. In blockoriented processing, these symbols must be known a-priori (pilots or null carriers). In contrast, block-adaptive processing utilizes symbol decisions, and channel estimation can benefit from signals received on all carriers.

An optimal solution to the channel estimation problem, be it of the least squares (LS), minimum mean squared error (MMSE), or maximum a-posteriori probability (MAP) type, involves a matrix inversion of size $M_{T} L$. This fact has motivated the development of channel estimation algorithms and pilot allocation strategies whose goal is to avoid matrix inversion or reduce its complexity.

Reduction in complexity has been sought through selection of significant impulse response coefficients which results in a reduced-size matrix inversion [7], [8], [6]. The adaptive algorithm [9] eliminates the need for matrix inversion by estimating each transmitter's response separately, having canceled the interference of other transmitter(s) using channel estimates from a previous block. This reference also provides optimal pilot sequences that simultaneously avoid matrix inversion and provide MMSE performance. The idea of decomposing the received signal into individual transmitters' contributions has further been explored in Ref. [10], where the expectationmaximization (EM) principle is used to arrive at the LS channel estimates in an iterative manner. The same MIMO-SIMO decomposition is utilized in a MAP channel estimator [11], which exploits low-rank approximation [12] to avoid matrix inversion. Computationally simple, but suboptimal algorithms can also be obtained by assuming the channel to be equal between adjacent carriers [13], [5], which results in $K$ parallel matrix inversions of size $M_{T}$. Channel estimation for MIMO systems that aim for diversity gain through space-time coding is addressed in Ref. [14].

In this paper, we adopt the framework of decision-directed adaptive block processing [6], making use of the Doppler compensation principle [3] and focusing on a least mean squares (LMS) channel estimator that does not require matrix inversion. The paper is organized as follows. After defining the system model in Sec. II, channel estimation is discussed in Sec. III. Sec. IV is devoted to performance illustration using real data transmitted over a $1 \mathrm{~km}$ shallow water channel in the $8-18 \mathrm{kHz}$ band. Concluding remarks are made in Sec. V. 


\section{SySTEM MOdEL}

The received signal after FFT demodulation is modeled as

$$
y_{k}^{r}(n)=\sum_{t=1}^{M_{T}} H_{k}^{t r}(n) d_{k}^{t}(n) e^{j \theta_{k}^{t}(n)}+z_{k}^{r}(n)
$$

where the indices $t, r, k, n$ refer to the transmitter, receiver, subband and time, respectively; $H$ refers to the channel, $z$ to the noise, and $d$ to the data symbols taken from an arbitrary PSK/QAM alphabet. The phase shift is modeled as

$$
\theta_{k}^{t}(n)=\theta_{k}^{t}(n-1)+a^{t}(n) \cdot 2 \pi f_{k} T^{\prime}
$$

where $f_{k}=f_{0}+k \Delta f$ is the $k$ th carrier frequency, $T^{\prime}=T+T_{g}$ is the time devoted to one OFDM block, which includes the signal of duration $T=1 / \Delta f$ and the multipath guard time $T_{g}$, and $a^{t}(n)$ represents the residual Doppler factor (after initial resampling), which is modeled as constant during one block, but allowed to vary from one block to another. ${ }^{1}$ Assuming that $a^{t}(n) f_{k}<<\Delta f, \forall t, k, n$, inter-carrier interference is treated as additional noise.

In matrix notation, the received signal (1) is given as

$$
\mathbf{y}_{k}(n)=\mathbf{d}_{k}(n) \boldsymbol{\Theta}_{k}(n) \mathbf{H}_{k}(n)+\mathbf{z}_{k}(n)
$$

where

$$
\begin{aligned}
\mathbf{y}_{k}(n) & =\left[y_{k}^{1}(n) \ldots y_{k}^{M_{R}}(n)\right] \\
\mathbf{d}_{k}(n) & =\left[d_{k}^{1}(n) \ldots d_{k}^{M_{T}}(n)\right] \\
\mathbf{z}_{k}(n) & =\left[z_{k}^{1}(n) \ldots z_{k}^{M_{R}}(n)\right]
\end{aligned}
$$

and

$$
\begin{aligned}
\mathbf{H}_{k}(n) & =\left[H_{k}^{t r}(n)\right]_{t=1, \ldots M_{T} ; r=1, \ldots M_{R}} \\
\boldsymbol{\Theta}_{k}(n) & =\operatorname{diag}\left[e^{j \theta_{k}^{t}(n)}\right]_{t=1, \ldots M_{T}}
\end{aligned}
$$

Given the channel matrices and the phases, the LS estimate of the data symbols transmitted on the $k$-th carrier is

$$
\hat{\mathbf{d}}_{k}(n)=\mathbf{y}_{k}(n) \mathbf{H}_{k}^{\prime}(n)\left[\mathbf{H}_{k}(n) \mathbf{H}_{k}^{\prime}(n)\right]^{-1} \boldsymbol{\Theta}_{k}^{*}(n)
$$

where the prime denotes conjugate transpose, and we are assuming that $M_{R} \geq M_{T}$. Since the channel and the phases are not known, their estimates will be used instead, and symbol decisions will be made by soft-decision decoding of the soobtained estimates.

\section{Channel Estimation}

Let us define the discrete Fourier relationship

$$
\mathbf{H}_{k}(n)=\sum_{l=0}^{K-1} \mathbf{h}_{l}(n) e^{-j 2 \pi k l / K}
$$

where the coefficients $\mathbf{h}_{l}(n)=\left[h_{l}^{t r}(n)\right]_{t=1, \ldots M_{T} ; r=1, \ldots M_{R}}$ represent the MIMO channel in the impulse response domain. Note that fewer than $K$ impulse response coefficients may suffice to represent all of the $K$ transfer function coefficients. In particular, we define $L$ as the total contiguous span, and $J \leq L$ as the number of significant impulse response coefficients. The number of significant coefficients is normally not

\footnotetext{
${ }^{1}$ Earlier experiments have shown that it suffices to use a single phase for multiple co-located receivers [3], [4], [5].
}

known a-priori, and neither are their positions $l_{j}, j=1, \ldots J$. However, their total extent $L$ is known from the expected multipath spread, which is a system design parameter in any OFDM system. Taking into account the fact that an underwater acoustic channel is rarely of minimum phase, we recognize that (10) can be re-written as

$$
\mathbf{H}_{k}(n)=\sum_{l=-A}^{L-1-A} \mathbf{h}_{l}(n) e^{-j 2 \pi k l / K}
$$

where $A \geq 0, \mathbf{h}_{0}(n)$ is taken as the reference tap, and it is understood that $\mathbf{h}_{l}(n)=\mathbf{h}_{K+l}(n)$ for negative values of $l$.

If we now form the matrices

$\mathbf{Y}(n)=\left[\begin{array}{l}\mathbf{y}_{0}(n) \\ \vdots \\ \mathbf{y}_{K-1}(n)\end{array}\right], \mathbf{D}_{\theta}(n)=\left[\begin{array}{l}\mathbf{d}_{0}(n) \boldsymbol{\Theta}_{0}(n) \\ \vdots \\ \mathbf{d}_{K-1}(n) \boldsymbol{\Theta}_{K-1}(n)\end{array}\right]$

and define $\boldsymbol{\Phi}=\operatorname{diag}\left[e^{-j 2 \pi k / K}\right]_{k=0, \ldots K-1}$, the received signals can be expressed in a compact form:

$$
\mathbf{Y}(n)=\sum_{l=-A}^{L-1-A} \boldsymbol{\Phi}^{l} \mathbf{D}_{\theta}(n) \mathbf{h}_{l}(n)+\mathbf{Z}(n)
$$

where the matrix $\mathbf{Z}(n)$ contains additive noise. This form serves as a basis for the design of the channel estimator.

For purposes of channel estimation, it is convenient to express the received signal as

$$
\mathbf{Y}(n)=\boldsymbol{\Delta}(n) \mathbf{h}(n)+\mathbf{Z}(n)
$$

where

$$
\boldsymbol{\Delta}(n)=\left[\boldsymbol{\Phi}^{-A} \mathbf{D}_{\theta}(n) \ldots \boldsymbol{\Phi}^{L-1-A} \mathbf{D}_{\theta}(n)\right]
$$

and $\mathbf{h}(n)$ contains the corresponding terms $\mathbf{h}_{l}(n)$, i.e. those with indices $l=-A, \ldots L-1-A$.

If all the data symbols are known, the LS channel estimate can be obtained as

$$
\hat{\mathbf{h}}(n)=\left[\boldsymbol{\Delta}^{\prime}(n) \boldsymbol{\Delta}(n)\right]^{-1} \boldsymbol{\Delta}^{\prime}(n) \mathbf{Y}(n)
$$

In order for such a solution to exist, the necessary condition is that $K \geq M_{T} L$. This condition can be interpreted in several ways: (1) for a given number of carriers $K$, at most $K / M_{T}$ channel coefficients can be estimated; (2) for a given channel span $L$, at least $M_{T} L$ observations are needed, and (3) for given $K$ and $L$, at most $K / L$ data streams can be multiplexed.

If fewer than $K$ observations are used, as would be the case in a pilot-assisted block-oriented approach, then those rows of the matrices $\mathbf{Y}(n)$ and $\boldsymbol{\Delta}(n)$ that correspond to the pilot carriers will be isolated from the expression (13) to form a reduced set of $\left(P=M_{T} L\right)$ observations. The underlying data symbols ( $M_{T}$ per observation) have to be known. In contrast, if all the data symbols can be known, as it is the case in a decision-directed approach, it is advantageous to utilize all $K$ observations instead of $M_{T} L$ only.

The knowledge of data symbols rests on the ability to accurately estimate the channel, and the assumption that the channel does not change much from one OFDM block to another. Channel estimates from the previous block can then be used to make tentative symbol decisions that will in turn 
be used to update the channel estimate. Note that pilot tones can also be used to aid decision-directed operation, but their number $P$ need not be constrained by the channel length.

The complexity of the problem can be reduced through channel sparsing. Namely, if only the $J$ significant channel entries are kept, the channel estimation problem (15) can be re-defined using

$$
\underline{\Delta}(n)=\left[\boldsymbol{\Phi}^{l_{1}} \mathbf{D}_{\theta}(n) \ldots \boldsymbol{\Phi}^{l_{J}} \mathbf{D}_{\theta}(n)\right]
$$

which will yield a corresponding channel estimate $\underline{\hat{\mathbf{b}}}(n)$ that now contains only the terms $\hat{\mathbf{h}}_{l_{j}}(n), j=1, \ldots J$. The coefficients to be kept are determined as those whose magnitude exceeds some threshold, which can be set in reference to the strongest coefficient for each transmitter, e.g. as $\gamma=20 \%$.

\section{A. Adaptation}

Adaptive channel estimation is initialized by the full-size solution (15), setting the phase estimates to zero and using known data symbols for which the inverse $\left[\boldsymbol{\Delta}^{\prime}(0) \boldsymbol{\Delta}(0)\right]^{-1}$ can be pre-computed. ${ }^{2}$

An adaptive algorithm can for instance be obtained by exponential weighting of the past estimates with $\lambda \in(0,1)$,

$$
\underline{\hat{\mathbf{h}}}(n)=\lambda \underline{\hat{\mathbf{h}}}(n-1)+(1-\lambda)\left[\underline{\boldsymbol{\Delta}}^{\prime}(n) \underline{\boldsymbol{\Delta}}(n)\right]^{-1} \underline{\boldsymbol{\Delta}}^{\prime}(n) \mathbf{Y}(n)
$$

Such an algorithm was successfully applied to an experimental data set [6], in which a benign channel and the relatively small bandwidth $(2.4 \mathrm{kHz})$ allowed sufficient sparsing $(J=4)$ to warrant the matrix inversion. In general, however, one cannot count on such a situation. An alternative is then given by the LMS approach, which is based on the modeling equation (13), or its sparse counterpart,

$$
\mathbf{Y}(n)=\underline{\Delta}(n) \underline{\mathbf{h}}(n)+\mathbf{Z}(n)
$$

This model implies that $\underline{\Delta}(n)$ can be regarded as an input to a filter $\underline{\mathbf{h}}(n)$, whose output, in the presence of noise, is $\mathbf{Y}(n)$. Since both the input and the output are known, the filter can be estimated in the LMS manner as

$$
\underline{\hat{\mathbf{h}}}(n)=\underline{\hat{\mathbf{h}}}(n-1)+\mu \underline{\Delta}^{\prime}(n)[\mathbf{Y}(n)-\underline{\boldsymbol{\Delta}}(n) \underline{\hat{\mathbf{h}}}(n-1)]
$$

where $\mu$ is the step size. Since the initial value $\underline{\hat{\mathbf{h}}}(0)$ is computed exactly using the known training symbols, slow convergence of the LMS algorithm is not an issue.

Additional sparsing can also be performed by setting to zero those elements of $\underline{\hat{\mathbf{h}}}(n)$ whose magnitude is below some threshold. The so-obtained impulse response coefficients are finally used to compute the corresponding transfer function coefficients (11), which are in turn used for data detection (9).

\section{B. Phase tracking}

Phase tracking in a MIMO system is based on estimating the Doppler factors $a^{t}(n)$ for all transmitters. This method is a straightforward extension of the SIMO case [3]. Assuming

\footnotetext{
${ }^{2}$ Training symbols can also be chosen so as to optimize the estimator and simultaneously trivialize the inversion [9].
}

the existence of a previous estimate $\hat{a}^{t}(n-1)$ and the phase $\hat{\theta}_{k}^{t}(n-1)$, a prediction for the current block is made as

$$
\check{\theta}_{k}^{t}(n)=\hat{\theta}_{k}^{t}(n-1)+\hat{a}^{t}(n-1) \cdot 2 \pi f_{k} T^{\prime}, \forall k, t
$$

This phase is used together with the existing channel estimate $\hat{\mathbf{H}}_{k}(n-1)$ to estimate the data symbol à la (9) and make a tentative decision $\overline{\mathbf{d}}_{k}(n)$. Had the outdated phase $\hat{\theta}_{k}^{t}(n-1)$ been used instead, the resulting estimate, $\hat{d}_{k, \text { out }}^{t}(n)$, would contain a phase error, which is measured as

$$
\psi_{k}^{t}(n)=\left\langle\hat{d}_{k, \text { out }}^{t}(n) \bar{d}_{k}^{t *}(n)\right\rangle, \forall k, t
$$

This error reflects the incremental phase shift over the time interval $T^{\prime}$ between two OFDM blocks, and can thus be used to estimate the Doppler factor via the modeling equation (2). To do so, averaging is performed over all the carriers,

$$
\hat{a}^{t}(n)=\frac{1}{K} \sum_{k} \frac{\psi_{k}^{t}(n)}{2 \pi f_{k} T^{\prime}}
$$

If the Doppler distortion can be modeled as equal for all the transmitters, averaging can be performed over the transmit elements as well. Additional filtering can also be performed, e.g. as

$$
\hat{a}^{t}(n)=\alpha \hat{a}^{t}(n-1)+(1-\alpha) \frac{1}{K} \sum_{k} \frac{\psi_{k}^{t}(n)}{2 \pi f_{k} T^{\prime}}
$$

where $\alpha \in(0,1)$ accounts for the filter memory. Doppler factor estimates are now used to update the phases,

$$
\hat{\theta}_{k}^{t}(n)=\hat{\theta}_{k}^{t}(n-1)+\hat{a}^{t}(n) \cdot 2 \pi f_{k} T^{\prime}, \forall k, t
$$

which are in turn used to make final symbol decisions and to update the channel estimates.

\section{EXPERIMENTAL RESULTS}

The algorithm described above was successfully applied to the real data collected during two recent experiments. The first experiment was conducted in March 2008, in the Narragansett Bay, off the coast of Rhode Island, and involved acoustic transmissions in the $10-12.4 \mathrm{kHz}$ band. Reference [6] reports on the results of that experiment. The second experiment, on which we report here, was conducted in October 2008, south of the Martha's Vineyard island, off the coast of Massachusetts, and involved transmissions in the $10-18 \mathrm{kHz}$ acoustic band.

The geometry of the experiment is illustrated in Fig.1. The signals were transmitted from one element at a time, so that they could later be combined to mimic a varying number of transmitters (between 1 and 3).

Experimental signals were generated using 4PSK and 8PSK signals with a varying number of carriers $K$. Transmission was organized in frames, with each frame carrying a total of $N_{d}=$ $2^{14}$ data symbols in $N=N_{d} / K$ OFDM blocks. Table I lists the signal parameters. The factor $\beta$ in this table represents the raw bandwidth efficiency in symbols/second/Hz/transmitter,

$$
\beta=\frac{1}{1+B T_{g} / K}
$$

The effective bandwidth efficiency obtained with $M_{T}$ transmitters and a modulation level $M, R_{b} / B=M_{T} \beta \log _{2} M$, is listed in Table II. 


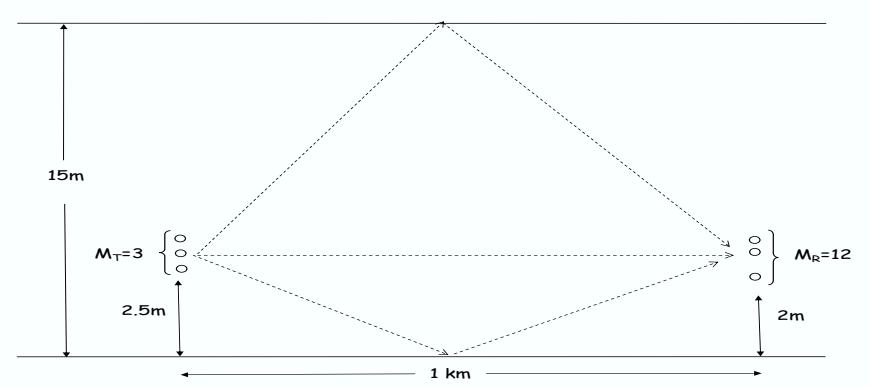

Fig. 1. Geometry of the experiment.

\begin{tabular}{|l||c|c|c|c|c|}
\hline OFDM(4,8-PSK) & $K$ & $N$ & $\Delta f$ & $T$ & $\beta$ \\
\hline \hline$B=10 \mathrm{kHz}$ & 128 & 128 & $78 \mathrm{~Hz}$ & $13 \mathrm{~ms}$ & 0.45 \\
\hline$T_{g}=16 \mathrm{~ms}$ & 256 & 64 & $39 \mathrm{~Hz}$ & $26 \mathrm{~ms}$ & 0.62 \\
\hline$f_{0}=8.25 \mathrm{kHz}$ & 512 & 32 & $19 \mathrm{~Hz}$ & $52 \mathrm{~ms}$ & 0.76 \\
\hline$N_{d}=2^{14}$ symb. & 1024 & 16 & $10 \mathrm{~Hz}$ & $105 \mathrm{~ms}$ & 0.86 \\
\hline
\end{tabular}

TABLE I

SIGNAL PARAMETERS USED IN THE EXPERIMENT.

The signals were also coded using the $\mathrm{BCH}(64,10)$ code. This code was chosen arbitrarily; a practical implementation could settle for a less powerful, more bandwidth-efficient one. Each string of 10 bits was encoded into a 32-symbol codeword for 4PSK (a 21-symbol codeword for 8PSK), and the codewords comprising one block were mapped onto the OFDM carriers so as to keep the symbols of the same codeword maximally separated in frequency. Confining the codewords to the same OFDM block does not exploit time diversity, but it enables instantaneous decoding for block-adaptive decisiondirected operation.

The signals were recorded over a period of two weeks, during which the weather conditions were varying, causing changes in the wave height, period and direction, as well as changes in the sound speed profile and other internal structures. These conditions influence the channel variability, and consequently the system performance. The overall system performance, as measured by the mean squared error (MSE) at the detector output, was observed to vary by a few $\mathrm{dB}$ depending on the particular conditions. In general, consistently good performance was observed at four different receiver locations (200 m and $1 \mathrm{~km}, \mathrm{SE}$ and SW from the transmitter) both with 4 and 8 PSK. We report here on a typical data set.

Fig. 2 shows the results of processing a $K=1024$ 4PSK frame corresponding to $M_{T}=3$ active transmitters (the results are shown for the data stream coming from the bottom transmit element). OFDM was implemented with zero padding, and overlap adding [15] was performed prior to FFT demodulation to take into account $3 \mathrm{~ms}$ of the guard interval before, and $7 \mathrm{~ms}$ after an OFDM block. This choice was made based on separate channel probing, which indicated a total multipath spread somewhat greater than $10 \mathrm{~ms}$. The number of impulse response coefficients is set to $L=128$ (13 ms). Shown in the figure is the raw received frame lasting $2 \mathrm{~s}$ (above); the phase estimates for several carriers (the corresponding Doppler factor is on the order of $10^{-5}$ ); the channel responses for all 12 receivers as seen at the end of the frame (note the two comparably strong

\begin{tabular}{|l||c|c|c|c|}
\hline$M_{T} \| K$ & 128 & 256 & 512 & 1024 \\
\hline \hline 1 & $0.9 / 1.3$ & $1.2 / 1.8$ & $1.5 / 2.3$ & $1.7 / 2.6$ \\
\hline 2 & $1.8 / 2.7$ & $2.5 / 3.7$ & $3.0 / 4.6$ & $3.5 / 5.2$ \\
\hline 3 & $2.7 / 4.0$ & $3.7 / 5.6$ & $4.6 / 6.9$ & $5.2 / 7.8$ \\
\hline
\end{tabular}

TABLE II

BANDWIDTH EFFICIENCY [BPS/HZ] OF THE EXPERIMENTAL 4PSK/8PSK SIGNALS, NOT COUNTING THE CODE RATE.

arrivals); the MSE in time (average over all carriers), and the MSE in frequency (average over all blocks). The overall MSE, taken as the average over all blocks and carriers, and the bit error rate (BER) measured over the frame, are indicated in the figure together with the various receiver parameters. Note that no pilots are used.

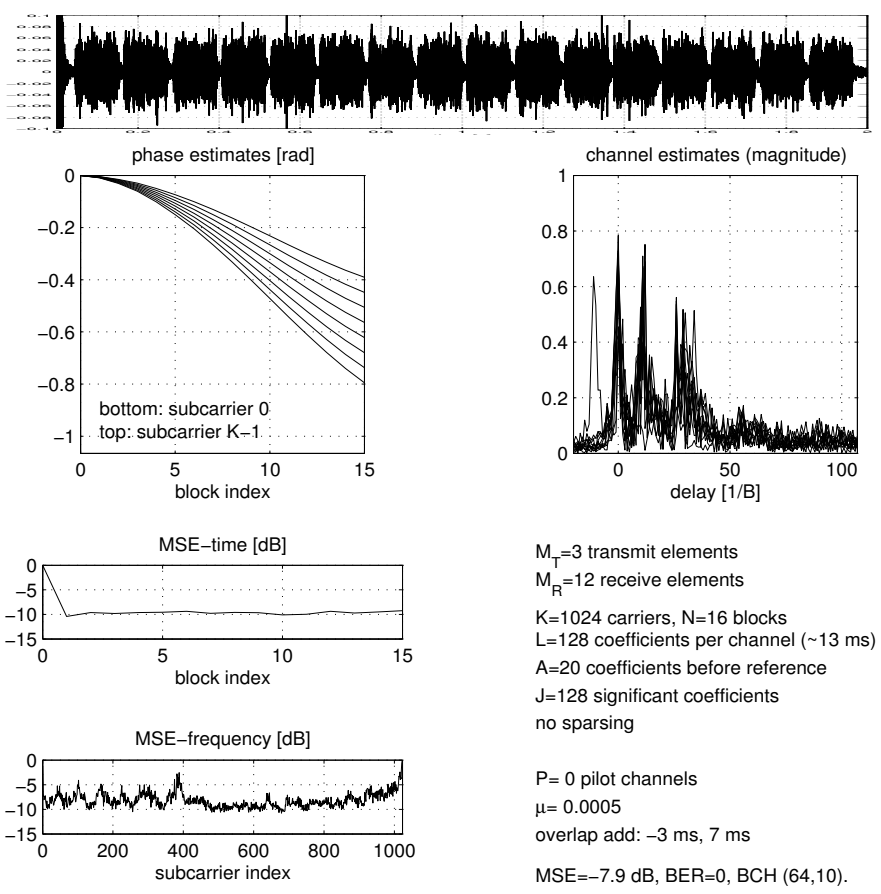

Fig. 2. Signal processing results $(10 / 23 / 2008,21: 53,1 \mathrm{~km}, \mathrm{SW})$.

Fig. 3 summarizes the results for this frame, obtained using varying system parameters. Shown in the figure is the overall MSE vs. the number of carriers $K$, for different number of transmitters $M_{T}$. For a single transmitter, the gradual degradation in performance with increasing $K$ is attributed to the increasing duration $T=K / B$ of the OFDM block, which allows a greater channel variation, thus making the tracking more difficult. When more than one transmitter is used, the estimated impulse response length is set to $L=\min \left\{\left\lfloor K / M_{T}\right\rfloor, 128\right\}$ to satisfy the requirement that $M_{T} L \leq K$. The effective multipath spread that can be captured by the estimator, $B K / M_{T}$, is listed in milliseconds in the table inset in Fig.3. We note that those $\left(M_{T}, K\right)$ pairs for which $B K / M_{T}<13$ ms coincide with the MSE above $-5 \mathrm{~dB}$. This MSE value also represents a threshold beyond which decision-directed operation ceases to be reliable. The initial decrease in the MSE with $K$ is thus attributed to an increasing number of observations available for the channel estimator. As $K$ reaches some point (256 for $M_{T}=2$ and 
512 for $M_{T}=3$ ) the estimator captures all of the channel response, and normal operation is established. From here on, the performance gradually degrades with a further increase in $K$, as longer block durations support a greater variation of the channel, similarly as in the single transmitter case. The performance of a MIMO system is ultimately limited by the ability to handle the crosstalk between channels, as evidenced by the fact that $M_{T}=3$ yields a higher MSE than $M_{T}=2$.

It may we worth noting at this point that although the MIMO MSE curves exhibit a minimum, the corresponding value of $K$ may not necessarily be the designer's best choice. A value greater than this may still keep the performance within prespecified limits, while offering better bandwidth efficiency. In general, that $\left(M_{T}, K\right)$ pair should be chosen for which the bandwidth efficiency is maximized while a pre-specified performance level is met.

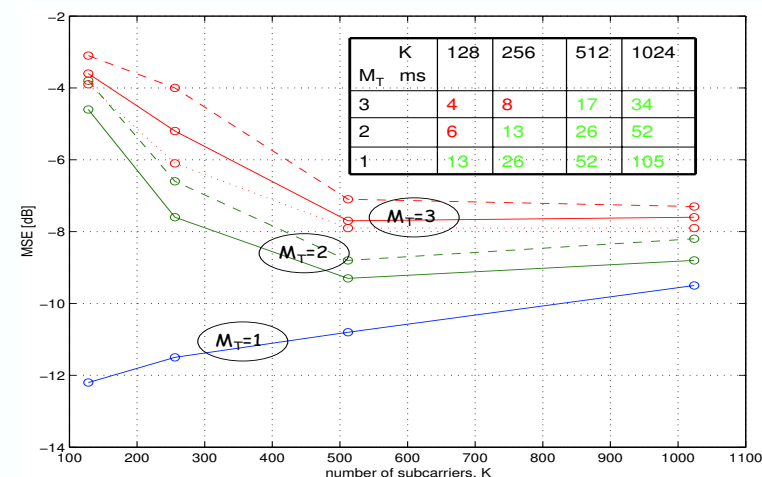

Fig. 3. Summary of signal processing result $(10 / 23 / 2008,21: 53,1 \mathrm{~km}, \mathrm{SW})$. Table shows the value $B K / M_{T}[\mathrm{~ms}]$.

\section{CONCLUSions}

Efficient use of acoustic bandwidth implies the need for a large number of carriers in an OFDM system, and multiple transmitters to support spatial multiplexing of data streams. However, the channel imposes limits on the system design. The number of carriers $K$ used in a fixed bandwidth $B$ is constrained by the coherence time of the channel, $T_{c o h}$, since conventional OFDM design, which is based on post-FFT processing and neglects the inter-carrier interference (ICI), rests on the assumption that the channel is (more or less) constant during one block, i.e. $T=K / B<<T_{\text {coh }}$. As the number of carriers increases, the block duration $T$ becomes longer, making it more difficult to track the channel (and eventually causing destructive ICI). The number of transmitters $M_{T}$ is at the same time constrained by the system's ability to resolve the crosstalk between parallel channels. To provide a sufficient number of observations for the channel estimator discussed in this paper, the number of transmitters is constrained to $M_{T} \leq K / L$, where $L$ is equal to the multipath spread $T_{m p}$ measured in $1 / B$. Together, these constraints imply a limit on the bandwidth efficiency, $R / B=M_{T} /\left(1+T_{m p} B / K\right) \leq$ $K^{2} / L(K+L)$. Ideally, the channel coherence time will be much greater than the multipath spread, $T_{c o h}>T_{m p}$, allowing efficient system design with $K>>L$. The bandwidth efficiency limit will then behave as $K / L$. This value, in turn, is constrained by the spread factor of the channel, $K / L<<T_{c o h} / T_{m p}$. It is important to note that the coherence time should be calculated so as to take into account only the time-variation seen by the post-FFT processor, and not the time-variation caused by the motion-induced Doppler effects that can be compensated for by resampling at the receiver's front end. This time variation includes the residual Doppler distortion after resampling (which we described by the factor a) and the inherent variation of the channel coefficients.

The receiver described in this paper combines adaptive channel estimation with non-uniform frequency offset compensation in a decision-directed algorithm that offers low computational complexity and low overhead. Experimental results demonstrate successful operation of a $3 \times 12 \mathrm{MIMO}$ system, using 4 and 8 PSK with 1024 carriers in a $10 \mathrm{kHz}$ acoustic bandwidth over $1 \mathrm{~km}$ in shallow water. These results serve as an encouragement for a real time implementation of MIMO OFDM in an acoustic modem.

\section{REFERENCES}

[1] B.Li, S.Zhou, M.Stojanovic, L.Freitag and P.Willet, "Multicarrier communications over underwater acoustic channels with nonuniform Doppler shifts," IEEE Journal of Oceanic Engineering, vol.33, No.2, April 2008, pp.198-209.

[2] B.Li, J.Huang, S.Zhou, K.Ball, M.Stojanovic, L.Freitag and P.Willett, "MIMO-OFDM for high rate underwater acoustic communications," IEEE Journal of Oceanic Engineering, to appear.

[3] M.Stojanovic, "Low complexity OFDM detector for underwater acoustic channels," in Proc. IEEE Oceans Conf., Sept. 2006.

[4] M.Stojanovic, "OFDM for underwater acoustic communications: adaptive synchronization and sparse channel estimation," in Proc. ICASSP, 2008.

[5] P.C.Ceballos and M.Stojanovic, "Adaptive MIMO detection of OFDM signals in an underwater acoustic channel," in Proc. IEEE Oceans Conf., 2008.

[6] M.Stojanovic, "Adaptive channel estimation for underwater acoustic MIMO OFDM Systems," in Proc. IEEE DSP/SPE Workshop, Jan. 2009.

[7] Y.Li, N.Seshadri and S.Ariyavisitakul, "Channel estimation for OFDM systems with transmitter diversity in mobile wireless channels," IEEE J.Select. Areas Commun., vol.17, No.3, March 1999, pp.461-471.

[8] H.Minn and V.Bhargava, "An investigation into time-domain approach for OFDM channel estimation," IEEE Trans. Broadcast., vol.46, No.4., Dec. 2000, pp.240-248.

[9] Y.Li, "Simplified channel estimation for OFDM systems with multiple transmit antennas," IEEE Trans. Wireless Commun., vol.1, No.1, Jan. 2002, pp.67-75.

[10] Y.Xie and C.Georghiades, "Two EM-type channel estimation algorithms for OFDM with transmitter diversity," IEEE Trans. Commun., vol.51, No.1, Jan. 2003, pp.106-115.

[11] J.Gao and H.Liu, "Low-complexity MAP channel estimation for mobile MIMO-OFDM systems," IEEE Trans. Wireless Commun., vol.7, No.3,March 2008, pp.774-780.

[12] O.Edfors, M.Sandell, J-J. ven de Beek, S.K.Wilson and P.O.Borjesson, "OFDM channel estimation by singular value decomposition,” IEEE Trans. Commun., vol.47, No.7, July 1998, pp.931-933.

[13] K.Minn, D.Kim and V.Bhargava, "A reduced complexity channel estimation for OFDM systems with transmit diversity in mobile wireless channels," IEEE Trans. Commun., vol.50, No.5, May 2002 pp.799-807.

[14] Y.Li, J.Winters and N.Sollenberger, "MIMO-OFDM for wireless communications: signal detection with enhanced channel estimation," IEEE Trans. Commun., vol.50, No.9, Sept. 2002, pp.1471-1477.

[15] B.Muquet, Z.Wang and G.Giannakis, "Cyclic prefix or zero padding for wireless multicarrier transmissions?," IEEE Trans. Commun., vol.50, No.12, Dec.2002, pp.2136-2148. 